\title{
Single-shot measurement of the Josephson charge qubit
}

\author{
O. Astafiev, ${ }^{1, \text { * }}$ Yu. A. Pashkin, ${ }^{1, \text { 由 T. Yamamoto, }}{ }^{1,2}$ Y. Nakamura,,${ }^{1,2}$ and J. S. Tsai ${ }^{1,2}$ \\ ${ }^{1}$ The Institute of Physical and Chemical Research (RIKEN), Wako, Saitama 351-0198, Japan \\ ${ }^{2}$ NEC Fundamental Research Laboratories, Tsukuba, Ibaraki 305-8501, Japan
}

(Dated: October 30, 2018)

\begin{abstract}
We demonstrate single-shot readout of quantum states of the Josephson charge qubit. The quantum bits are transformed into and stored as classical bits (charge quanta) in a dynamic memory cell - a superconducting island. The transformation of state $|1\rangle$ (differing form state $|0\rangle$ by an extra Cooper pair) is a result of a controllable quasiparticle tunneling to the island. The charge is then detected by a conventional single-electron transistor, electrostatically decoupled from the qubit. We study relaxation dynamics in the system and obtain the readout efficiency of $87 \%$ and $93 \%$ for $|1\rangle$ and $|0\rangle$ states, respectively.

PACS numbers: 03.67.-a, 74.50.+r, 85.25.Cp
\end{abstract}

It has been recently realized that Josephson junctions can be used for building quantum bits (qubits) and integrated quantum computer circuits controlled by external electrical signals 1, 2]. After the first experiments on single Josephson qubits 3, 4, 5, 6, 7], an important step towards the integration has been made: coherent control of two qubits and conditional gate operation have been experimentally demonstrated for two electrostatically coupled charge qubits [8, 9]. However, in these experiments, individual probabilities of each qubit averaged over all states of the other qubit were measured 10]. To directly measure multi-qubit states, one must be able to readout each qubit after every single-shot coherent state manipulation. The single-shot readout is of great importance, for instance, for quantum state tomography, quantum state teleportation, quantum cryptography [11]. Without the single-shot readout, algorithms that give non-unique solutions can not be utilized.

To readout single quantum states of the Josephson qubits (in particular, flux qubits) through the phase degree of freedom, a few circuits, measuring switching event from the supercurrent state to the finite-voltage state were implemented [4, 5, 6, 7]. In charge type of qubits, it is straightforward to measure a charge quantum instead of the flux quantum 12]. For the single-shot charge readout, a radio-frequency single-electron transistor [13] electrostatically coupled to the qubit was proposed as a detector of the charge states 14, 15]. Although this approach works in principle [16, 17], the single-shot readout has not yet been realized. In this work, we demonstrate an operation and study mechanism of novel readout scheme that allows to perform highly efficient singleshot measurements, with suppressed back-action of the measurement circuit on the qubit.

A scanning-electron micrograph of our circuit is shown in Fig. 11(a) The device consists of a charge qubit [3] and a readout circuit. The qubit is a Cooper-pair box (with

*Electronic address: astf@frl.cl.nec.co.jp

†On leave from Lebedev Physical Institute, Moscow 117924, Russia its effective capacitance to the ground $C_{b} \approx 600 \mathrm{aF}$ ) coupled to a reservoir through a Josephson junction with the Josephson energy $E_{J} \approx 20 \mu \mathrm{eV}$. The reservoir is a big island with about $0.1 \mathrm{nF}$ capacitance to the ground plane and galvanically isolated from the external environment. The qubit states are coherently controlled by a non-adiabatic control pulse, yielding a superposed state of $|0\rangle$ and $|1\rangle$. The readout part includes an electrometer which is a conventional low-frequency single-electron transistor $(\mathrm{SET})\left(C_{s} \approx 1000 \mathrm{aF}\right)$ and a charge trap $\left(C_{t} \approx\right.$ $1000 \mathrm{aF}$ ) placed between the qubit and the SET. The trap is connected to the box through a highly resistive tunnel junction $\left(R_{t} \approx 100 \mathrm{M} \Omega\right)$ and coupled to the SET with a capacitance $C_{s t} \approx 100 \mathrm{aF}$. The use of the trap enables us to separate in time the coherent state manipulation and readout processes and, in addition, the qubit becomes electrostatically decoupled from the SET. The qubit relaxation rate induced by the SET voltage noise is suppressed by a factor of $\left(C_{b t} C_{s t} / C_{t} C_{b}\right)^{2} \approx 3 \times 10^{-5}$, where $C_{b t} \approx 30 \mathrm{aF}$ [18]. The coupling strength can be made even weaker, if the unwanted box-to-trap capacitance $C_{b t}$ is further decreased.

The operation of the circuit can be described in the following way. During the qubit manipulation, the trap is kept unbiased prohibiting charge relaxation to the trap. Once the control pulse is terminated, the readout pulse (see the inset of Fig. 1(a)) is applied to the trap. The length and the amplitude of the readout pulse are adjusted so that if there is an extra Cooper pair in the box after termination of the control pulse, it escapes to the trap through a quasiparticle tunneling with a high probability. After the charge is trapped, it remains in the trap for a long time (a reverse trap-to-box charge relaxation is suppressed due to the superconducting energy gap 2 $\Delta$ ) and is measured by a low-frequency SET.

The Hamiltonian of the two-level system of the qubit in the charge basis $|0\rangle$ and $|1\rangle$ (without and with an extra Cooper pair $)$ is $H=U_{b}\left(0, Q_{b}\right)|0\rangle\left\langle 0\left|+U_{b}\left(2, Q_{b}\right)\right| 1\right\rangle\langle 1|$ $1 / 2 E_{J}(|0\rangle\langle 1|+| 1\rangle\langle 0|$ ) (we define an electrostatic energy of island $k$ as $U_{k}\left(N_{k}, Q_{k}\right)=\left(N_{k} e-Q_{k}\right)^{2} / 2 C_{k}$, where $k$ is either $b$ or $t$ indicating box or trap island, respectively, $N_{k}$ is an excess electron number, and $Q_{k}$ is a gate in- 

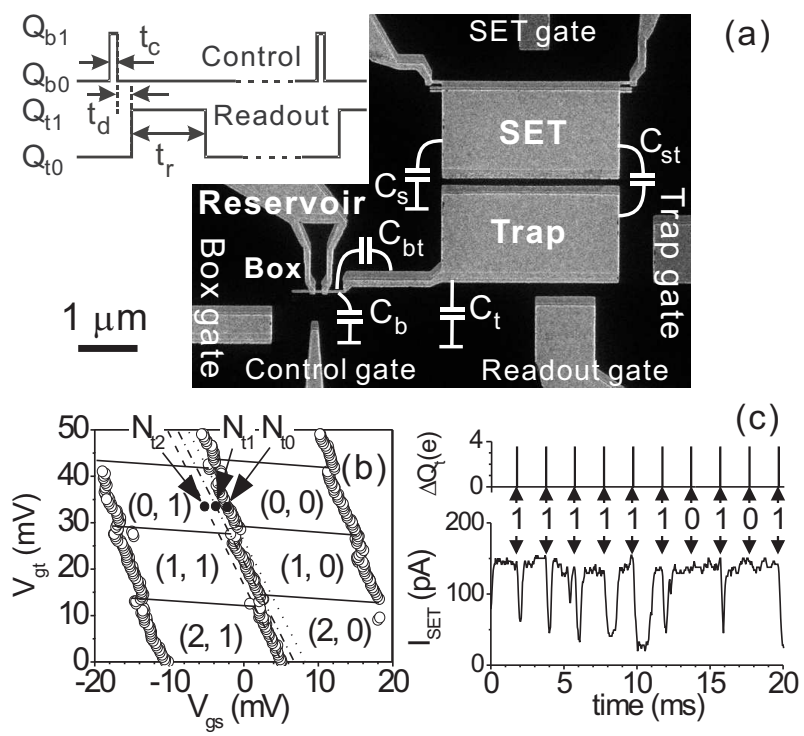

FIG. 1: (a) Scanning electron micrograph of the device. The aluminum structure is deposited on top of a thin $\mathrm{Si}_{3} \mathrm{~N}_{4}$ insulating layer $(0.4 \mu \mathrm{m})$ above a gold ground plane. The device consists of a Cooper pair box, a reservoir, a trap and a measurement SET. The dc ("box" and "trap") and pulse (control and readout) gates control potentials of the islands. Pulse operation is schematically represented in the inset. (b) Stability diagram of the SET coupled to the trap. Open circles mark positions of the SET quasiparticle current peaks on $V_{g s}-V_{g t}$ plane (dc gate voltages of the SET and the trap). Pairs of numbers $\left(N_{t}, N_{s}\right)$ designate the trap - SET ground state charge configuration in each cell bounded by the SET peaks and solid lines. Dashed, dashed-dotted and dotted lines indicate positions of the SET peaks for 0,1 or 2 additional electrons in the trap, respectively. (c) A typical time-trace of the SET current (lower panel) together with the readout pulse sequence (upper panel). Negative switches on the lower curve correspond to the detected charge of the trap. Digits 1 and 0 mark readout bits.

duced charge in the island. Starting at $Q_{b 0}\left(Q_{b 0}<Q_{b 1}\right)$, where $E>>E_{J}\left(\Delta E=U_{b}\left(2, Q_{b}\right)-U_{b}\left(0, Q_{b}\right)\right)$ we let the system relax to the ground state, which is nearly pure charge state $|0\rangle$. Then we instantly change the eigenbasis for a time $t_{c}$ by applying a rectangular control pulse, which brings the system to $Q_{b 1}$. If $Q_{b 1}$ is a degeneracy point $(\Delta E=0)$, the final state of the control pulse manipulation is $|0\rangle \cos \omega_{J} t_{c} / 2+|1\rangle \sin \omega_{J} t_{c} / 2\left(\omega_{J}=E_{J} / \hbar\right)$, therefore, after the pulse termination, the state $|1\rangle$ is realized with a probability of $\cos ^{2} \omega_{J} t_{c} / 2$.

Fig. 1(b) shows an experimentally measured stability diagram: SET current peak positions as a function of trap and box gate voltages of the SET and the trap. By setting the box and trap gates to one of the points $N_{t 0}, N_{t 1}$ or $N_{t 2}$, we can detect if the trap has 0,1 or 2 additional electrons. In our measurements, the SET is usually adjusted to $N_{t 0}$. To readout the qubit, the trap is biased by the readout pulse of typical length $t_{r}=300 \mathrm{~ns}$ and amplitude $\Delta Q_{t}=3.5 e\left(\Delta Q_{t}=Q_{t 1}-Q_{t 0}\right)$, applied to the readout gate, letting an extra Cooper pair of the state

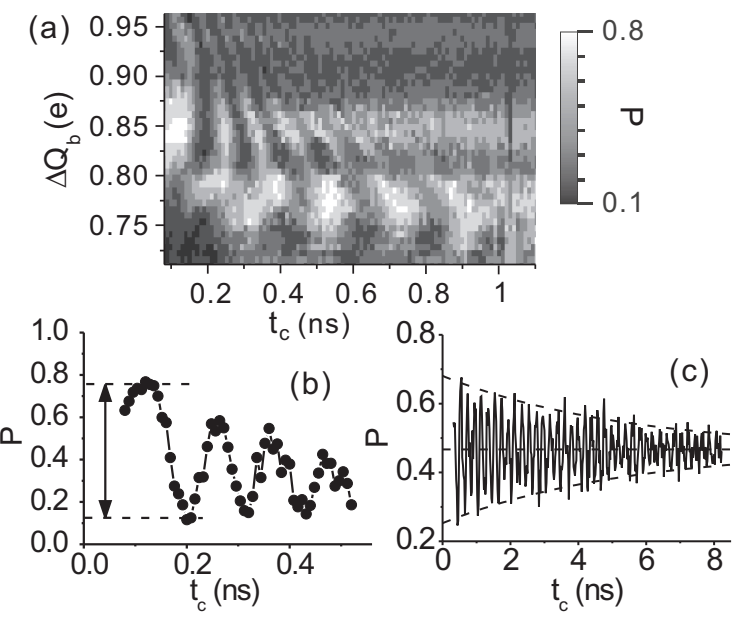

FIG. 2: (a) Coherent oscillations measured by averaging over many events of the single-shot measurements. (b) $P$ versus $t_{c}$ measured at $\Delta Q_{b}=0.84 e\left(\equiv Q_{b A}\right)$, where visibility is the highest. (c) $P$ versus $t_{c}$ measured at $Q_{b}=0.75 e$ $\left(\equiv Q_{b B}\right)$, where the oscillations are the longest lasting (degeneracy point). Dashed envelops correspond to the exponential decay with the decay time of $5.8 \mathrm{~ns}$.

$|1\rangle$ escape to the trap through a quasiparticle tunneling and switching off the SET current (the SET peak position is shifted to the position of the dashed line).

The curve in the upper panel of Fig. 1(c) indicates a readout pulse sequence. The curve on the lower panel demonstrates a typical time-trace of the SET current. Negative switches on the curve of the lower panel coming synchronously with the readout pulses are counted as charge detection events. For the studied device, the lifetime of the trapped charge is typically about $300 \mu \mathrm{s}$, therefore, normally used repetition time of $2 \mathrm{~ms}$ is sufficient for practically complete trap resetting. We count the number of detected switches $m$, with the total number of shots $n_{t}$.

An experimentally obtained probability of the charge detection $P=m / n_{t}$ (normally, $n_{t}=327$ is used per one experimental data point) as a function of the control pulse length $t_{c}$ and the amplitude $\Delta Q_{b}\left(\Delta Q_{t}=\right.$ $\left.Q_{t 1}-Q_{t 0}\right)$ is shown as a two-dimensional plot in Fig.2 (a). We define the pulse with $\Delta Q_{b}=0.84 e\left(\equiv Q_{b A}\right)$ and $t_{c}=120 \mathrm{ps}$, when $P$ reaches maximum, as a $\pi$-pulse. Fig. 2(b) demonstrates coherent oscillations as a function of $t_{c}$ measured at $\Delta Q_{b A}$. As shown by the vertical arrowed line, the visibility here reaches 0.64 , while the longest lasting oscillations shown in Fig. 2(c) are found at $\Delta Q_{b}=0.75 e\left(\equiv Q_{b B}\right)$, (the phase decoherence is expected to be the weakest at the degeneracy point). We believe that $Q_{b A} \neq Q_{b B}$ due to the control pulse distortion because of limited frequency bandwidths of the transmission lines and the pulse generator.

Curves with open and closed dots in Figs. 3(a-b) represent $P$ as a function of the readout pulse amplitude $\Delta Q_{t}$ with and without control $\pi$-pulses, respectively. The 

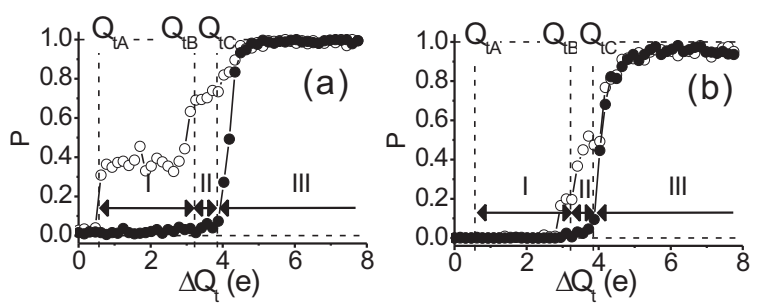

FIG. 3: $\quad P$ versus readout pulse amplitude $\Delta Q_{t}$ with and without $\pi$-pulse (open and closed cycles, respectively). (a) $P$ measured at $N_{t 0}$ (see Fig. 1(b)). (b) $P$ measured at $N_{t 2}$. One may distinguish three different regions on the plots: (I) a finite probability for the excited state detection is only at $N_{t 0}$ position; (II) finite probabilities for the excited state detection are at $N_{t 0}$ and $N_{t 2}$ positions; (III) switches are detected even if the qubit is in the state $|0\rangle$. Threshold amplitudes $Q_{t A}, Q_{t B}, Q_{t C}$ are derived for the box-to-trap relaxation processes $(2,0) \rightarrow(1,1),(1,1) \rightarrow(0,2)$ and $(0,0) \rightarrow(-1,1)$, respectively.

probabilities measured at $N_{t 0}$ are shown in Fig. 31(a), while the probabilities measured at $N_{t 2}$ (where positive switches are counted) are shown in Fig. B(b). One may divide the plots into three regions marked by I, II and III differing from each other by the counting characteristics. Based on the data from these plots, we suppose that when the qubit is in the state $|1\rangle$, one quasiparticle tunnels from the box to the trap in the process $(2,0) \rightarrow$ $(1,1)$ within the region I $\left(\left(N_{b}, N_{t}\right)\right.$ represents the boxtrap quasiparticle configuration); quasiparticles tunnel to the trap in the process $(2,0) \rightarrow(1,1) \rightarrow(0,2)$ within the region II; a quasiparticle tunneling process becomes possible even for the state $|0\rangle$ in the region III.

The quasiparticle tunneling is energetically feasible in the process $\left(N_{b}, N_{t}\right) \rightarrow\left(N_{b}-1, N_{t}+1\right)$, when the following condition is satisfied $U_{b}\left(N_{b}, Q_{b 0}\right)+U_{t}\left(N_{t}, Q_{t 1}\right)>$ $U_{b}\left(N_{b}-1, Q_{b 0}\right)+U_{t}\left(N_{t}+1, Q_{t 1}\right)+2 \Delta$ [19] (we neglect the interaction energy term which is as small as the interisland coupling strength). Substituting an explicit expression for the energies, one may find the necessary trap readout pulse amplitude for the quasiparticle escape $\Delta Q_{t}>Q_{t}^{\prime}+N_{t} e+\eta\left(2-N_{b}\right) e$, where $\eta=C_{t} / C_{b}$ and $Q_{t}^{\prime}=e / 2-\eta\left(3 e / 2-Q_{b 0}\right)+2 \Delta C_{t} / e-Q_{t 0}$. We define three threshold amplitudes of $\Delta Q_{t}$, at which the following processes become possible: $(2,0) \rightarrow(1,1)$ at $\Delta Q_{t}>Q_{t A}$, where $Q_{t A}=Q_{t}^{\prime} ;(1,1) \rightarrow(0,2)$ at $\Delta Q_{t}>Q_{t B}$, where $\Delta Q_{t B}=Q_{t}^{\prime}+(1+\eta) e ;(0,0) \rightarrow(-1,1)$ at $\Delta Q_{t}>Q_{t C}$, where $Q_{t C}=Q_{t}^{\prime}+2 \eta e$. The threshold amplitudes calculated with $Q_{t A}=0.6 e$ (taken from the first step of $P$ curve on Fig. 3(a)) and $\eta=1.67$ are $Q_{t B}=3.3 e$ and $Q_{t C}=3.9 e$ and shown on the Figs. 31(a-b) by the dashed vertical lines. Note that the probability for the trap to have a charge at $\Delta Q_{t}>4.5 e$ reaches 1 in Fig. 3(a). This proves that once the charge is trapped it is detected with $100 \%$ probability. For the highest efficiency, we set the operation point of the SET to $N_{t 0}$, where either one or two trapped quasiparticles yield a negative switch of the

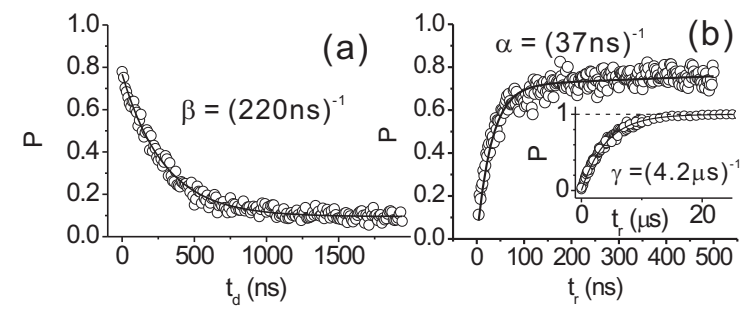

FIG. 4: (a) $P$ as a function of delay between the control $\pi$ pulse and the readout pulse $t_{d}$. A solid curve is a fitting by an exponent with a decay rate $\beta=(220 \mathrm{~ns})^{-1}$. (b) A probability of $|1\rangle$-states detection created by the -pulse as a function of the readout pulse length $t_{r}$. A solid curve is a result of fitting $m(t)$ using Eq. (1) normalized by $n_{t}$ with fitting parameters $n_{0}^{*}$ and $\alpha\left(n_{0}^{*} / n_{t}=0.87\right.$ and $\left.\alpha=(37 \mathrm{~ns})^{-1}\right)$. The inset shows probability without $\pi$-pulses. $P$ is fitted by $1-\exp \left(-\gamma t_{r}\right)$ with $\gamma=(4.1 \mu \mathrm{s})^{-1}$.

SET current and the pulse amplitude to $\Delta Q_{t} \approx 3.5 e$, at which two quasiparticles may escape to the trap.

Figs. 4 (a-b) demonstrate time relaxation dynamics of the qubit states. Fig. 世(a) shows a probability $P$ to find an extra charge in the trap, when time delay $t_{d}$ is introduced between the control $\pi$-pulse and the readout pulses. The exponential decay of $P$ may be explained by tunneling to the reservoir (presumably via energetically feasible Cooper pair tunneling $(2,0) \rightarrow(0,0)$ ) because alternative quasiparticle relaxation to the trap through the high resistive junction is blocked by $2 \Delta$ when the trap is not biased. The relaxation rate to the reservoir found from the fitting (solid curve) is $\beta=(220 \mathrm{~ns})^{-1}$. Fig. 4 (b) shows relaxation dynamics of the state $|1\rangle$ as a function of the readout pulse length $t_{r}\left(t_{d} \approx 0\right)$. This relaxation is mainly determined by quasiparticle decay to the trap with a rate $\alpha(\alpha \gg \beta)$. Additionally, the inset of Fig. 4(b) shows dynamics of $|0\rangle$-state relaxation ("dark" switches) to the trap. These "dark" switches can be presumably described by the process $(0,0) \rightarrow(-2,2)$, with a weak relaxation rate $\mu=(4100 \mathrm{~ns})^{-1}$ derived from fitting the data by $1-\exp \left(-\gamma t_{r}\right)$ (solid curve).

Let us consider the relaxation dynamics in more details. The number of excited states, $n^{*}$, decreases within the time interval $[t, t+d t]$ as $d n^{*}(t)=-\alpha n^{*}(t) d t-$ $\beta n^{*}(t) d t$ 20]. The number of states in $(0,0)$ configuration, $n(t)$, changes, in turn, as $d n(t)=-\gamma n(t) d t+$ $\beta n^{*}(t) d t$. We may also write an expression for the number of events in which the trap is found to be charged: $d m(t)=n^{*}(t) d t+n(t) d t$. Solving these equations with the initial conditions $n^{*}(0)=n_{0}^{*}, n(0)=n_{t}-n_{0}^{*}$ and $m(0)=0$ we find

$$
m(t)=n_{t}\left[1-e^{-\gamma t}\right]+\frac{n_{0}^{*}(\alpha-\gamma)}{\alpha+\beta-\gamma}\left[e^{-\gamma t}-e^{-(\alpha+\beta) t}\right] .
$$

We fit the data of Fig. 4(b) by a curve of $P=m(t) / n_{t}$ with $m(t)$ taken from Eq. (1) with two fitting parameters $\alpha$ and $n_{0}^{*}$. The fitting gives $\alpha=(37 \mathrm{~ns})^{-1}[21]$ and $n_{0}^{*} / n_{t}=0.84$, implying that the efficiency of $|0\rangle$-to- $|1\rangle$ 
conversion by the control $\pi$-pulse is $84 \%$.

If our readout pulse length $t=t_{r}$ satisfies the conditions $\gamma \ll t_{r}^{-1} \ll \alpha+\beta$, then Eq. (1) can be simplified to

$$
m(t) \approx n_{0}^{*} \frac{\alpha}{\alpha+\beta}+\gamma t_{r}\left[n_{t}-n_{0}^{*} \frac{\alpha}{\alpha+\beta}\right] .
$$

Using Eq. (2), one may estimate an efficiency of the single-shot readout. We introduce a probability $P_{y}(x)$ of finding the trap charged $(y=1)$ or uncharged $(y=$ $0)$, when the qubit is in $|x\rangle$-state ( $x$ is either 0 or 1 ). According to the definition of $P_{y}(x), P_{0}(0)+P_{1}(0)=1$ and $P_{0}(1)+P_{1}(1)=1$. The total number of detected events expressed in terms of these probabilities may be written as $m=n_{0}^{*} P_{1}(1)+\left(n_{t}-n_{0}^{*}\right) P_{1}(0)$. Comparing the latter expression with Eq. (2) we find

$$
\begin{array}{r}
P_{1}(1)=\frac{\alpha+\beta \gamma t_{r}}{\alpha+\beta} \\
P_{1}(0)=\gamma t_{r} .
\end{array}
$$

Confirming that our readout pulse length $t_{r}=300 \mathrm{~ns}$ fulfills the necessary condition for Eqs. (3), $\gamma \ll t_{r}^{-1} \ll$ $\alpha+\beta$, we directly find from Eqs. (3) that the probability of detection of the state $|1\rangle$ is $P_{1}(1)=0.87$ and the state $|0\rangle$ is $P_{0}(0)=0.93\left(P_{1}(0)=0.07\right)$. The readout efficiency can be further improved by optimizing the relaxation rates. The derived probabilities are consistent with the mean probability of the oscillations at the degeneracy point, $\langle P\rangle=\left[\left\langle n_{0}^{*}\right\rangle P_{1}(1)+\left(n_{t}-\left\langle n_{0}^{*}\right\rangle\right) P_{1}(0)\right] / n_{t}=0.47$ (horizontal line in Fig. 4( $(\mathrm{c})$ ), where $\left\langle n_{0}^{*} / n_{t}\right\rangle=0.5$.

Finally, we would like to briefly discuss new possibilities and potential applications, which this scheme opens. The trap island works as a dynamic memory cell, which stores the multi-qubit state in a classical bit. Therefore, the data can be further processed in a classical way. For example, implementing sequential readout of many cells connected in series, similarly to that of charge-coupled devices will allow to reduce a number of output circuit channels.

One of straightforward applications of the described device, staying outside quantum computation direction, is a tunable single-photon detector in a centimeter wavelength range. A weak microwave radiation may resonantly excite the box near the degeneracy point from the ground to the excited state with a finite probability. The excited state may then be converted into the charge state $|1\rangle$ by an adiabatic sweep of the control gate voltages (a relatively slow gate voltage change forces the system to move along eigenenergy bands) and detected by the single-shot measurement circuit.

As for the nearest plans, it is straightforward to use the single-shot readout to for measuring double qubit system [8, 9]. This will allow us to demonstrate entangled states and controlled-NOT gate operation.

We thank S. Lloyd for valuable discussion.
[1] Y. Makhlin, G. Schon, and A. Shnirman, Rev. Mod. Phys. 73, 357 (2001).

[2] D. V. Averin, Fortshr. Rev. 48, 1055 (2000).

[3] Y. Nakamura, Y. A. Pashkin, and J. S. Tsai, Nature 398, 786 (1999).

[4] D. Vion, A. Aassime, A. Cottet, P. Joyez, H. Pothier, C. Urbina, D. Esteve, and M. H. Devoret, Science 296, 886 (2002).

[5] Y. Yu, S. Han, X. Chu, S. I. Chu, and Z. Wang, Science 296, 889 (2002).

[6] J. M. Martinis, S. Nam, J. Aumentado, and C. Urbina, Phys. Rev. Lett. 89, 117901 (2002).

[7] I. Chiorescu, Y. Nakamura, C. J. P. M. Harmans, and J. E. Mooij, Science 299, 1869 (2003).

[8] Y. A. Pashkin, T. Yamamoto, O. Astafiev, Y. Nakamura, and J. S. Tsai, Nature 421, 823 (2003).

[9] T. Yamamoto, Y. A. Pashkin, O. Astafiev, Y. Nakamura, and J. S. Tsai, Nature 425, 941 (2003).

[10] Quasiparticle currents induced in the measurement circuits of the qubits are proportional to individual probabilities of finding the qubits in the state $|1\rangle$ (with one extra Cooper pair). That is, if the wave-function in the charge basis of the double qubit basis of $\left|i_{1} i_{2}\right\rangle$ is $\sum_{i_{1} i_{2}} C_{i_{1} i_{2}}\left|i_{1} i_{2}\right\rangle\left(i_{k}\right.$ are 1 or 0 depending on whether the qubit $k$ has an extra Cooper pair or not), the current induced, for example, in the qubit 1 measurement circuit is proportional to $\sum_{i_{2}}\left|C_{i_{1} i_{2}}\right|^{2}$.

[11] M. A. Nielsen and I. L. Chuang, Quantum Computa- tion and Quantum Information. (Cambridge Univ. Press, Cambridge, 2000).

[12] A.Shnirman and G. Schon, Phys. Rev. B57, 15400 (1998).

[13] R. J. Schoelkopf, P. Wahlgren, A. A. Kozhevnikov, P. Delsing, and D. E. Prober, Science 280, 1232 (1998).

[14] A. Aassime, G. Johansson, G. Wendin, R. J. Schoelkopf, and P. Delsing, Phys. Rev. Lett. 86, 3376 (2001).

[15] G. Johansson, A. Käck, and G. Wendin, Phys. Rev. Lett. 88, 046802 (2002).

[16] T. Duty, D. Gunnarsson, K. Bladh, R. J. Schoelkopf, and P. Delsing (2002), cond-mat/0305433.

[17] K. W. Lehnert, K. Bladh, L. F. Spietz, D. Gunnarson, D. I. Schuster, P. Delsing, and R. J. Schoelkopf, Phys. Rev. Lett. 90, 027002 (2003).

[18] We estimate the qubit energy relaxation rate during coherent state manipulation induced by our measurement SET to be lower than $(0.5 \mu \mathrm{s})^{-1}$.

[19] In our case of the high resistive junction and relatively short readout pulses, an additional energy $2 \Delta$ is needed for quasiparticle tunneling independently of parity because of low tunneling rate of an unpaired quasiparticle.

[20] We simplify this problem avoiding consideration of the detailed relaxation mechanism and assume that $n^{*}$ includes any $\left(N_{b}, 0\right)$ charge configurations with $N_{b} \neq 0$. Most importantly, the experimentally measured decay of $n^{*}$ in Fig. 4 (a) is perfectly exponential.

[21] The tunnel junction resistance is estimated as $R_{t} \approx$ $2 \Delta / \alpha e^{2}=100 \Omega \mathrm{M}$. 